\title{
La pollution des eaux de la zone urbaine d'une lagune tropicale par les matières oxydables (lagune Ebrié, Côte d'Ivoire)
}

\author{
K. M. YAO ${ }^{1}$, B. S. METONGO ${ }^{1}$, A. TROKOUREY ${ }^{2 *}$ et Y. BOKRA ${ }^{2}$ \\ ${ }^{1}$ Centre de Recherches Océanologiques (CRO), 29 rue des Pêcheurs, BP V 18 Abidjan, Côte d'Ivoire. \\ ${ }^{1}$ Centre de Recherches Océanologiques (CRO), 29 rue des Pêcheurs, BP V 18 Abidjan, Côte d'Ivoire. \\ ${ }^{2}$ Laboratoire de Chimie Physique, Université de Cocody, 22 BP 582 Abidjan 22, Côte d'Ivoire. \\ ${ }^{2}$ Laboratoire de Chimie Physique, Université de Cocody, 22 BP 582 Abidjan 22, Côte d'Ivoire. \\ *Auteur correspondant, E-mail : trokourey@yahoo.com
}

\section{RESUME}

Suite à l'explosion démographique et au déversement de déchets hautement toxiques dans la métropole d'Abidjan, la qualité physico-chimique de cinq baies fermées de la lagune Ebrié en face d'Abidjan a été évaluée de 2006 à 2008. Plusieurs paramètres (température, pH, salinité, oxygène dissous, matières oxydables et sels nutritifs) ont été mesurés. Il apparaît que les quatre premières baies, soumises aux rejets d'eaux résiduaires, possèdent de très fortes charges en particules en suspension, $\mathrm{DBO}_{5}$, et $\mathrm{DCO}$, et sont le siège de mortalités de la faune aquatique et d'une accumulation de sédiments riches en matières organiques. En revanche, la cinquième, éloignée des rejets, a enregistré des concentrations deux à trois fois plus faibles ; elle est bien oxygénée et présente des signes d'un dépôt des particules minérales. Les résultats des teneurs en sels nutritifs révèlent que les teneurs en ammonium de certaines zones affectent la vie aquatique et que les principales sources de sels nutritifs sont les rejets domestiques et industriels, les eaux de ruissellement et l'érosion des sols du bassin versant du fleuve Comoé. Ces résultats permettront de dépolluer les baies de la lagune Ebrié et de surveiller l'utilisation des engrais dans la région d'Abidjan.

(C) 2009 International Formulae Group. All rights reserved.

Mots clés: Pollution, lagune, matières oxydables, nutriments.

\section{INTRODUCTION}

La plupart des lagunes africaines subissent une détérioration accrue de leur qualité ces dernières décennies. Cette dégradation est due au développement conjoint de l'urbanisation et des activités industrielles et agricoles. La lagune Ebrié qui constitue le plus grand système lagunaire de l'Afrique occidentale n'est pas en reste. La détérioration de la qualité de ses eaux est devenue un problème complexe, aussi bien quant aux conditions écologiques, qu'en ce qui concerne le développement socioéconomique de la ville d'Abidjan qu'elle entoure. Cette détérioration est associée aux déversements abusifs d'eaux résiduaires rejetées dans le milieu naturel sans aucun traitement préalable (Arfi et al., 1981; Haskoning, 1999). Ces apports de composés biodégradables causent d'intenses phénomènes d'eutrophisation, en particulier dans les zones à faible taux de renouvellement (Pagès et al., 1980; Zabi, 1982). Ce milieu et les ressources qui lui sont associées constituent un enjeu national majeur en matière d'économie et de santé publique. Dans l'état actuel des connaissances, la principale source de pollution de la lagune est d'ordre organique (Guiral et al., 1984; Yao et al., 2007) et provient essentiellement des eaux usées domestiques et industrielles, raccordées ou non au réseau d'égouttage. Cependant, le 
problème majeur qui se pose demeure la quantification globale basée sur la mesure directe de la pollution organique de la lagune Ebrié. Les informations qui en découlent sont indispensables pour lutter contre la dégradation du milieu. De plus, la qualité physico-chimique des eaux lagunaires entourant la ville d'Abidjan pourrait être modifiée par l'explosion démographique qu'elle connaît depuis la guerre de 2002 et par le déversement de déchets hautement toxiques en septembre 2006 dans la région.

L'objectif de cette étude est d'évaluer la qualité physico-chimique des baies fermées de la lagune Ebrié, soumises à des rejets d'Abidjan, par l'estimation saisonnière des teneurs en matières oxydables et en sels nutritifs et par l'évaluation de leurs variations dans les différents compartiments du milieu (dissous et particulaire). Cette caractérisation permettra la mise à jour de la qualité physicochimique des eaux lagunaires d'Abidjan.

\section{MATERIEL ET METHODES}

Situation géographique et stations de prélèvements

La lagune Ebrié, avec une superficie de $566 \mathrm{~km}^{2}$ (Varlet, 1978), s'étire sur $125 \mathrm{~km} \mathrm{le}$ long du littorale de la Côte d'Ivoire, entre $3^{\circ} 40^{\prime}$ et $4^{\circ} 50^{\prime}$ Ouest, à la latitude de $5^{\circ} 50^{\prime}$ Nord (figure 1). Le volume de cette lagune est d'environ $2,510^{9} \mathrm{~m}^{3}$, la profondeur moyenne est de $4,8 \mathrm{~m}$ et quelques fosses proches d'Abidjan dépassent $20 \mathrm{~m}$. La lagune Ebrié est séparée de l'Océan Atlantique par un cordon littoral sableux de 1 à $8 \mathrm{~km}$, traversé en sa partie centrale par le canal de Vridi depuis 1951. Ce canal, large de $300 \mathrm{~m}$ et profond environ une vingtaine de mètres est la seule voie de communication avec la mer.

Notre étude s'est portée sur la partie estuarienne typique en face d'Abidjan (Figure 1 ), en raison de l'accentuation de la pollution dans diverses baies fermées de cette zone. La lagune Ebrié est alimentée par les eaux de l'océan, au travers du canal de Vridi, en saison sèche (décembre à mai), et par des cours d'eau douce, le principal, le fleuve Comoé pendant la saison des crues, de septembre à novembre. Les précipitations sont réparties en deux saisons de pluies, la grande se situant entre fin mai et juillet tandis que la petite est centrée sur octobre (Figure 2). Selon
Varlet (1978), les apports d'eau douce représentent annuellement plus de 4 fois le volume total de la lagune tandis que les entrées d'eau marine sont quatorze fois plus importantes que ce même volume. Une description topographique, hydroclimatique et chimique plus précise est faite par Varlet (1978), Durant et Skubisch (1979) et Pagès et al. (1979).

La ville d'Abidjan s'étend sur ses berges avec une population évaluée à 3,6 millions d'habitants en 2002. La plupart des industries du pays y sont concentrées. Les problèmes de pollutions sont causés par des apports excessifs sans traitement préalables d'effluents domestiques et industriels (savonneries, huileries, brasseries, laiteries, raffineries...). Cette zone est aujourd'hui considérée comme un réservoir de bactéries pathogènes.

Dix neuf stations de prélèvement ont été retenues dans cinq baies de la zone estuarienne de la Lagune Ebrié. Les baies de Biétri, Banco, Cocody, et Marcory sont soumises à des rejets abusifs d'effluents domestiques et industriels. En revanche, la baie des Milliardaires, est éloignée des rejets directs (Figure 1). Plusieurs critères ont guidé nos choix notamment, l'urbanisation et la proximité du canal de Vridi.

\section{Echantillonnage et mesures in situ}

Les campagnes de mesures ont été réalisées tous les deux mois de juillet 2006 à avril 2008. Les prélèvements d'eau sont effectués à $0,5 \mathrm{~m}$ de la surface et du fond, au moyen d'une bouteille Niskin dans des flacons en polyéthylène de $1 \mathrm{~L}$ pour les analyses au laboratoire. Immédiatement après la remontée de la bouteille à bord de l'embarcation, les mesures in situ de certains paramètres physico-chimiques ont été faites. L'eau a été ensuite recueillie, tout en filtrant sur une toile de nylon de $200 \mu \mathrm{m}$ de maille, chaque échantillon dans les flacons de prélèvement à travers la soupape de vidange. Le $\mathrm{pH}$, la température et la salinité ont été mesurés à l'aide d'un multi paramètre de type WTW pH/cond 340 i. L'oxygène dissous a été déterminé par une sonde à DBO YSI 58 et par la méthode de Winkler (1888); la transparence par la plongée du disque de Secchi. Les échantillons ont été acheminés au laboratoire 




Figure 1: Localisation de la zone d'étude et stations de prélèvement

à l'obscurité, dans une glacière à $4{ }^{\circ} \mathrm{C}$ et conservés au congélateur à $-20^{\circ} \mathrm{C}$

\section{Analyse des échantillons au laboratoire}

Le traitement a débuté dès le retour au laboratoire et s'est étendu sur trois jours, afin de tenir compte du délai de conservation pour chaque type d'analyse. Les orthophosphates $\left(\mathrm{PO}_{4}{ }^{3-}\right)$ ont été déterminés par la méthode de Murphy et Riley (1962), les nitrites $\left(\mathrm{NO}_{2}{ }^{-}\right)$et les ions ammonium $\left(\mathrm{NH}_{4}^{+}\right)$par dosage colorimétrique selon la méthode de Koroleff (1976) décrite par Aminot et Chaussepied (1983). 


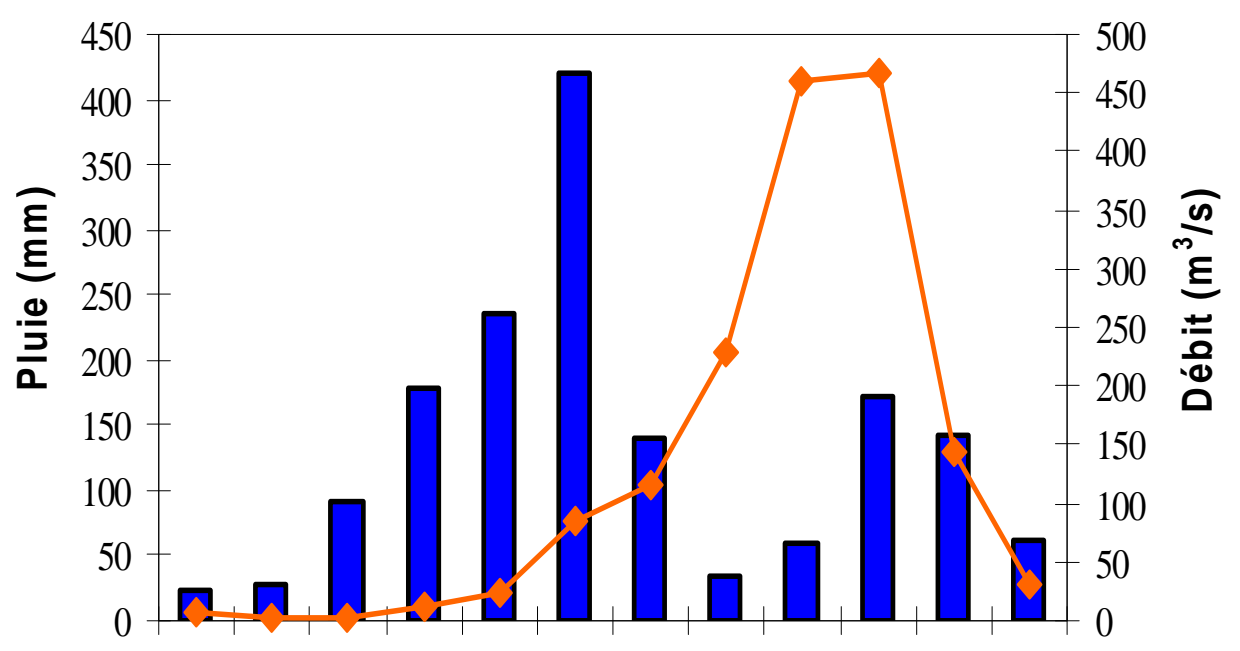

jan. fév. mars avril mai juin juil. août sept. oct. nov. déc.

\section{Mois}

$\square$ Pluie $\leadsto$ Débit

Figure 2 : Moyennes mensuelles des débits du fleuve Comoé et de la pluviométrie de 1982 à 2006. [Source de données: Société d'Exploitation et de développement aéroportuaire Aéoronautique Météorologique (SODEXAM) et Direction de l'Hydraulique Humaine (DHH)].

La Demande Biochimique en Oxygène $\left(\mathrm{DBO}_{5}\right)$ correspondant à une estimation de la charge organique biodégradable a été déterminée par la méthode MA.315-DBO 1.0 modifiée du Centre d'Expertise en Analyse Environnementale du Québec (édition 02-031999), après une bonne homogénéisation de l'échantillon. Cette méthode consiste à déterminer la quantité d'oxygène consommée par la matière oxydable à l'aide de bactéries acclimatées pour une période de cinq jours d'incubation à $20{ }^{\circ} \mathrm{C}$. Afin d'équilibrer la quantité de matière oxydable et d'oxygène disponible, un volume approprié d'échantillons est placé dans une bouteille en verre de $300 \mathrm{ml}$ en présence de bactéries et de substances nutritives. La concentration de l'oxygène dissous est mesurée par dosage selon la méthode de Winkler (1888) au début et à la fin de la période d'incubation. La quantité d'oxygène consommée est proportionnelle à la concentration de matières oxydables.

La Demande Chimique en Oxygène (DCO) est déterminée par la méthode au dichromate de potassium selon la norme AFNOR NF T90-101 après une forte homogénéisation de l'échantillon pour les échantillons non filtrés. Une correction de chlorures a été faite par ajout de $10 \mathrm{mg}$ de sulfate de mercure (HgSO4) par $\mathrm{mg}$ de chlorure présent dans l'échantillon. Pour caractériser les flux des polluants, la distribution entre phase dissoute $(<0,43 \mu \mathrm{m})$ et particulaire $(>0,43 \mu \mathrm{m})$ a été déterminée.

Les Matières en Suspension (MES) ont été mesurées selon la méthode décrite par Rodier (1996).

\section{Analyses statistiques}

La variabilité spatio-temporelle des différents paramètres a été étudiée par l'analyse des variances (ANOVA) à $\mathrm{P}<0,05$. La comparaison multiple des moyennes des concentrations ainsi que leur classement ont été réalisés par le test de Newman-Keuls chaque fois que l'analyse de variance a révélé des différences significatives. Tous les calculs statistiques ont été faits à l'aide du logiciel Statistica 7.1. 


\section{RESULTATS}

Ordre de grandeur des paramètres physico-chimiques

Les moyennes cumulées des paramètres physico-chimiques mesurées dans les baies (2006-2008) sont indiquées dans le tableau 1.

Les températures moyennes des baies se situent entre $28,97 \pm 1,50{ }^{\circ} \mathrm{C}$ et $29,74 \pm 1,50$ ${ }^{\circ} \mathrm{C}$ en surface, alors qu'à l'interface eausédiment, les eaux sont plus froides et comprises entre $26,86 \pm 2,46{ }^{\circ} \mathrm{C}$ et $28,81 \pm 1,25$ ${ }^{\circ} \mathrm{C}$. Les amplitudes des variations spatiales et verticales sont faibles (1 à $\left.2{ }^{\circ} \mathrm{C}\right)$. Aucune variation spatiale significative n'a été observée pour les eaux de surface $(\mathrm{P}<0,05)$.

La gamme des moyennes de $\mathrm{pH}$ des cinq baies étudiées est comprise entre $7,46 \pm 0,53$ pour la baie de Marcory et $8,17 \pm 0,71$ pour la baie de Biétri en surface avec une faible dispersion des valeurs, indiquée par les écart-types (Tableau 1). A l'interface eau-sédiment, les valeurs sont plus élevées ; la moyenne minimale a été observée en baie de Marcory $(7,58 \pm 0,46)$ alors que le maximum a été enregistré en baie des
Milliardaires $(7,89 \pm 0,53)$. Les $\mathrm{pH}$ des eaux des baies du Banco, Biétri et Milliardaires proches des eaux marines sont significativement plus élevés que ceux de Cocody et Marcory pour les eaux de surface $(\mathrm{P}<0,05)$.

Les valeurs moyennes maximales de salinité ont été observées dans les baies de Biétri et des Milliardaires qui sont proches du canal de Vridi. Les eaux sont plus salées en profondeurs qu'en surface et l'amplitude verticale augmente avec la profondeur des stations.

La transparence moyenne des baies est faible ; elle varie entre $0,71 \pm 0,25 \mathrm{~m}$ (baie du Banco) et 1,90 $\pm 0,64 \mathrm{~m}$ (baie des Milliardaires). Les moyennes des baies de Cocody, Marcory, Banco et Biétri ne sont pas significativement différentes les unes des autres, mais elles sont significativement inférieures à celles de la baie des milliardaires à $\mathrm{P}<0,05$.

$$
\text { Les concentrations moyennes }
$$

d'oxygène dissous observées en surface sont très faibles dans la baie de Cocody $(4,04 \pm 2,75$ $\mathrm{mg} / \mathrm{L})$ et de Marcory $(3,85 \pm 2,66 \mathrm{mg} / \mathrm{L})$

Tableau 1 : Caractéristiques physico-chimiques des eaux à la surface (S) et à l'interface eausédiment (F) des baies de la lagune Ebrié.

\begin{tabular}{|c|c|c|c|c|c|c|}
\hline Paramètres & & Banco & Cocody & Marcory & Milliardaires & Biétri \\
\hline \multirow{2}{*}{$\mathrm{T}\left({ }^{\circ} \mathrm{C}\right)$} & $S$ & $29,72 \pm 1,66$ & $28,97 \pm 1,50$ & $29,11 \pm 1,60$ & $29,13 \pm 1,09$ & $29,74 \pm 1,50$ \\
\hline & $\mathrm{F}$ & $26,86 \pm 2,46$ & $28,38 \pm 1,74$ & $28,23 \pm 2,08$ & $27,45 \pm 2,68$ & $28,81 \pm 1,25$ \\
\hline \multirow{2}{*}{$\mathrm{pH}$} & $S$ & $7,90 \pm 0,51$ & $7,52 \pm 0,38$ & $7,46 \pm 0,53$ & $8,06 \pm 0,55$ & $8,17 \pm 0,71$ \\
\hline & $\mathrm{F}$ & $7,87 \pm 0,35$ & $7,69 \pm 0,42$ & $7,58 \pm 0,46$ & $7,89 \pm 0,53$ & $7,73 \pm 0,63$ \\
\hline \multirow{2}{*}{$\begin{array}{l}\text { Salinité } \\
(\% \circ)\end{array}$} & $\mathrm{S}$ & $14,92 \pm 8,90$ & $14,26 \pm 9,01$ & $14,34 \pm 8,83$ & $18,85 \pm 10,22$ & $16,48 \pm 9,57$ \\
\hline & $\mathrm{F}$ & $25,02 \pm 10,14$ & $18,30 \pm 10,54$ & $18,85 \pm 10,80$ & $24,76 \pm 10,74$ & $29,71 \pm 6,34$ \\
\hline \multirow{2}{*}{$\begin{array}{l}\mathrm{O}_{2} \\
(\mathrm{mg} / \mathrm{L})\end{array}$} & $\mathrm{S}$ & $6,59 \pm 3,13$ & $4,04 \pm 2,75$ & $3,85 \pm 2,66$ & $6,60 \pm 1,49$ & $6,70 \pm 2,83$ \\
\hline & $\mathrm{F}$ & $1,76 \pm 1,88$ & $3,43 \pm 2,08$ & $3,22 \pm 2,54$ & $4,12 \pm 2,49$ & $1,56 \pm 2,29$ \\
\hline Transp (m) & & $0,71 \pm 0.25$ & $0.80 \pm 0,38$ & $0,78 \pm 0,32$ & $1,90 \pm 0,64$ & $0,80 \pm 0,35$ \\
\hline \multirow{2}{*}{$\begin{array}{l}\mathrm{DBO}_{5} \\
(\mathrm{mg} / \mathrm{L})\end{array}$} & $\mathrm{S}$ & $111,7 \pm 47,8$ & $112,0 \pm 48,5$ & $101,4 \pm 41,7$ & $51,7 \pm 22,7$ & $125,6 \pm 64,4$ \\
\hline & $\mathrm{F}$ & $140,88 \pm 65,19$ & $128,36 \pm 54,58$ & $111,2 \pm 40,2$ & $97,49 \pm 26,62$ & $145,1 \pm 64,2$ \\
\hline \multirow{2}{*}{$\begin{array}{l}\mathrm{DCO} \\
(\mathrm{mg} / \mathrm{L})\end{array}$} & $\mathrm{S}$ & $286,3 \pm 107,8$ & $273,7 \pm 95,8$ & $309,7 \pm 157,2$ & $175,50 \pm 60,51$ & $381,8 \pm 130,3$ \\
\hline & $\mathrm{F}$ & $375,2 \pm 125,7$ & $346,7 \pm 110,3$ & $353,7 \pm 137,8$ & $327,5 \pm 138,3$ & $456,8 \pm 147,3$ \\
\hline \multirow{2}{*}{$\begin{array}{l}\text { MES } \\
(\mathrm{mg} / \mathrm{L})\end{array}$} & $S$ & $48,40 \pm 23,51$ & $38,78 \pm 19,50$ & $36,39 \pm 19,80$ & $29,97 \pm 16,35$ & $43,46 \pm 21,45$ \\
\hline & $\mathrm{F}$ & $57,84 \pm 24,68$ & $45,47 \pm 17,36$ & $41,10 \pm 17,59$ & $49,62 \pm 28,37$ & $50,76 \pm 32,01$ \\
\hline \multirow{2}{*}{$\begin{array}{l}\mathrm{NO}_{2}^{-}{ }^{-} \\
(\mu \mathrm{mol} / \mathrm{L})\end{array}$} & $\mathrm{S}$ & $4,76 \pm 4,34$ & $3,49 \pm 2,74$ & $4,01 \pm 3,08$ & $2,22 \pm 3,39$ & $3,31 \pm 3,47$ \\
\hline & $\mathrm{F}$ & $1,83 \pm 2,09$ & $2,94 \pm 1,98$ & $3,69 \pm 3,01$ & $0,96 \pm 1,11$ & $2,37 \pm 2,69$ \\
\hline \multirow{2}{*}{$\begin{array}{l}\mathrm{NH}_{4}^{+} \\
(\mu \mathrm{mol} / \mathrm{L})\end{array}$} & $\mathrm{S}$ & $12,58 \pm 14,21$ & $26,30 \pm 32,13$ & $11,91 \pm 9,48$ & $2,22 \pm 2,39$ & $12,09 \pm 21,49$ \\
\hline & $\mathrm{F}$ & $13,90 \pm 14,05$ & $20,15 \pm 21,75$ & $15,40 \pm 14,87$ & $1,60 \pm 2,14$ & $20,09 \pm 16,63$ \\
\hline \multirow{2}{*}{$\begin{array}{l}\mathrm{PO}_{4}^{3-} \\
(\mu \mathrm{mol} / \mathrm{L})\end{array}$} & $\mathrm{S}$ & $3,34 \pm 3,81$ & $4,36 \pm 3,13$ & $4,02 \pm 3,05$ & $2,79 \pm 5,25$ & $3,72 \pm 4,30$ \\
\hline & $\mathrm{F}$ & $3,97 \pm 3,44$ & $4,20 \pm 3,28$ & $4,45 \pm 4,19$ & $3,59 \pm 4,38$ & $6,84 \pm 8,20$ \\
\hline
\end{tabular}


alors qu'elles sont de $6,60 \mathrm{mg} / \mathrm{L}$ dans les autres baies. A l'interface eau-sédiment, les eaux sont anoxiques avec des valeurs inférieures à $2 \mathrm{mg} / \mathrm{L}$ dans la plupart des baies, sauf en baie des Milliardaires où la teneur moyenne est de 4,12 $\pm 2,49 \mathrm{mg} / \mathrm{L}$. Deux groupes homogènes ont été révélés pour les eaux de surface : le premier, caractérisé par de faibles teneurs, est formé par les baies de Marcory et Cocody tandis que le second, plus oxygéné, rassemble les baies de Biétri, Banco, et Milliardaires.

Les valeurs de $\mathrm{DBO}_{5}$ sont très élevées dans la colonne d'eau. En surface, la moyenne de $\mathrm{DBO}_{5}$ varie de $51,68 \pm 22,69 \mathrm{mg} \mathrm{O}_{2} / \mathrm{L}$ en baie des Milliardaires à $125,62 \pm 64,44 \mathrm{mg}$ $\mathrm{O}_{2} / \mathrm{L}$ en baie de Biétri. A l'interface eausédiment, elle se situe entre $97,49 \pm 26,62 \mathrm{mg}$ $\mathrm{O}_{2} / \mathrm{L}$ (baie des Milliardaires) et $145,16 \pm 64,21$ $\mathrm{mg}_{2} / \mathrm{L}$ (baie de Biétri). Anova a révélé pour les eaux de surface, une différence significative entre la baie des Milliardaires et les baies du Banco, Cocody, Marcory, et Biétri qui forment un groupe homogène.

Les valeurs minimales de DCO sont observées en baie des Milliardaires tandis que les valeurs maximales sont enregistrées en baie de Biétri.

Les eaux sont très chargées en matières en suspension; la valeur moyenne minimale est observée en baie des Milliardaires $(29,97 \pm 16,35 \mathrm{mg} / \mathrm{L})$ et la valeur maximale a été enregistrée à l'interface eau-sédiment de la baie du Banco $(57,84 \pm 24,68 \mathrm{mg} / \mathrm{L})$. Une différence significative a été observée entre la baie des Milliardaires et les baies du Banco et de Biétri pour les eaux de surface; en revanche, aucune différence significative n'a été observée entre les quatre baies et celle des Milliardaires dans la couche profonde.

\section{Caractéristiques des particules en suspension}

La teneur en matières organiques des particules a été évaluée au moyen des rapports MVS/MES (mg/mg), $\mathrm{DBO}_{5}$ particulaire/MES (mg $\mathrm{O}_{2} / \mathrm{mg}$ ) et DCO particulaire/MES $\left(\mathrm{mg} \mathrm{O}_{2} / \mathrm{mg}\right)$. Les matières en suspension possèdent de fortes valeurs en matières organiques volatiles et connaissent des fluctuations très marquées (Figure 3). Les baies du Banco, Cocody, Marcory et Biétri forment un groupe homogène dans la couche profonde, tandis que la baie des Milliardaires se distingue d'elles par des valeurs plus faibles. Les $\mathrm{DBO}_{5}$ liées aux particules en suspension dans la baie de Marcory sont significativement plus élevées que celles des autres baies. Les teneurs en DCO des particules en suspension dans les baies sont très élevées et ne sont pas statistiquement différentes.

La valeur moyenne varie de 0,13 à 0,26 pour le pourcentage de $\mathrm{DBO}_{5}$ particulaire $\left(\mathrm{DBO}_{5} \mathrm{p} / \mathrm{DBO}_{5}\right)$ et de 0,17 à 0,30 pour le pourcentage de DCO particulaire (DCOp/DCO) en surface (Figure 3). Dans les baies du Banco, Cocody, Marcory et Biétri, la proportion de matière organique sous forme particulaire vaut le double de celle de la baie des Milliardaires. Le pourcentage de $\mathrm{DBO}_{5}$ particulaire des baies du Banco, Cocody, Marcory et Biétri est significativement plus élevé que celui des Milliardaires.

Les teneurs moyennes en nitrites et en orthophosphates ont été partout inférieures à 5 $\mu \mathrm{mol} / \mathrm{L}$. Les teneurs en ammonium ont varié entre $2,22 \pm 2,39 \mu \mathrm{mol} / \mathrm{L}$ et $26,30 \pm 32,13$ $\mu \mathrm{mol} / \mathrm{L}$. Cependant, les concentrations en ammonium peuvent localement dépasser 111 $\mu \mathrm{mol} / \mathrm{L}$. C'est le cas des stations 5 et 6 de la baie de Cocody et 15 de la baie de Biétri (Figure 4).

\section{Variations saisonnières}

La température a affiché une variation saisonnière significative en surface comme en profondeur avec des valeurs plus élevées pendant la saison sèche. La salinité diminue significativement de la saison sèche à la saison des crues dans toutes les baies (Figure $5)$.

Dans l'ensemble, les teneurs en oxygène dissous chutent pendant la saison des pluies, surtout au fond de la baie de Biétri (Figure 5). Les eaux de l'interface eausédiment sont restées sous-saturées toutes les saisons. Les variations saisonnières de la $\mathrm{DBO}_{5}$ des eaux de surface dépendent de la baie. Dans les baies du Banco et de Cocody, les plus fortes valeurs ont été enregistrées en saison des pluies alors que la baie de Biétri a affiché les valeurs maximales pendant la saison des crues $(\mathrm{P}<0,05)$. Une différence significative a été observée pour la DCO des eaux de surface entre les saisons 


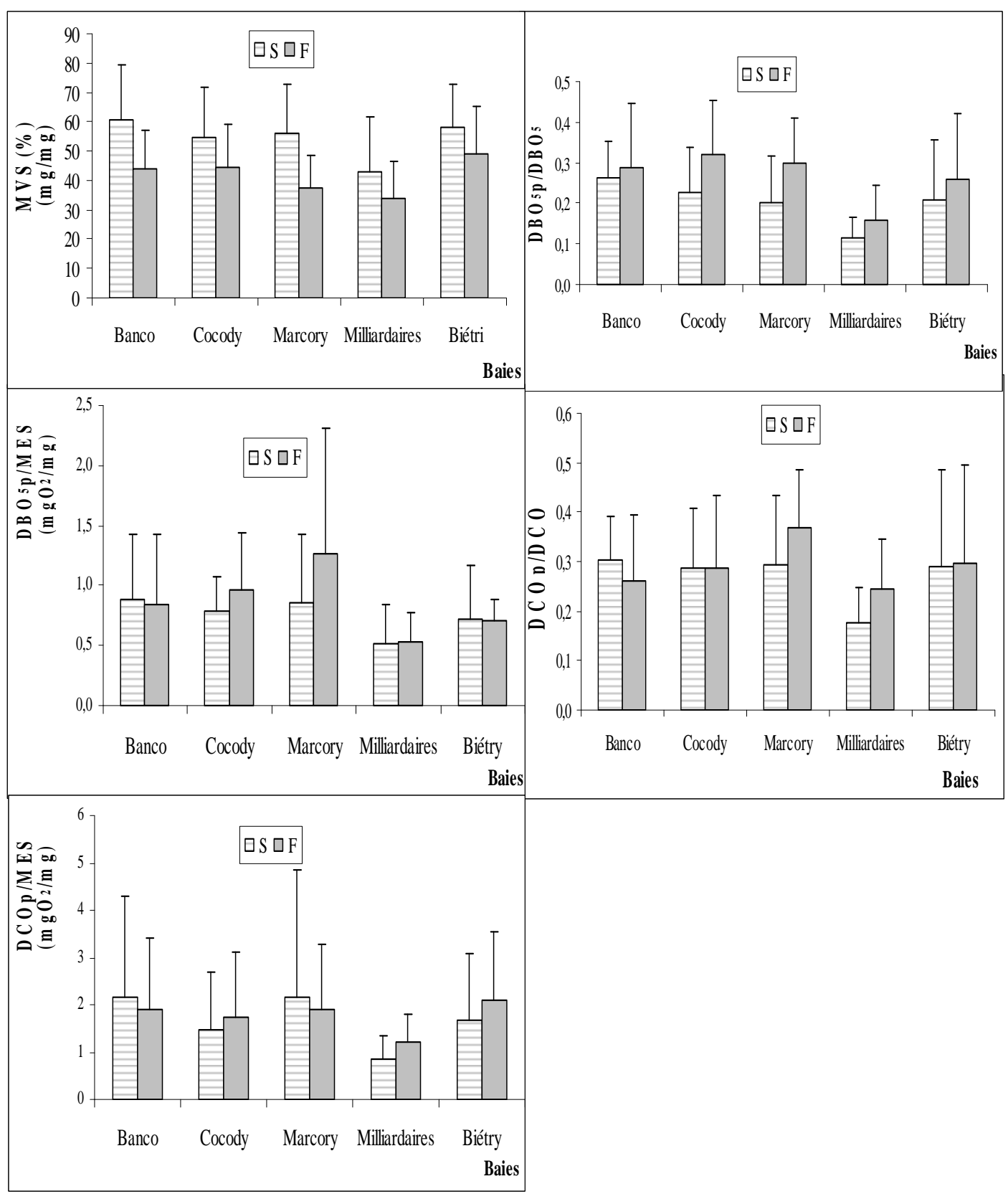

Figure 3 : Caractéristiques des particules en suspension à la surface $(\mathrm{S})$ et à l'interface eausédiment $(\mathrm{F})$ des baies de la lagune Ebrié. DCOp : DCO particulaire; $\mathrm{DBO}_{5} \mathrm{p}$ : $\mathrm{DBO}_{5}$ particulaire ; DCO : DCO totale ; $\mathrm{DBO}_{5}: \mathrm{DBO}_{5}$ totale. Les barres verticales représentent les écarts types.

des crues et de pluies contre la saison sèche. Dans les eaux de fond, les moyennes de MES atteignent leur maximum en saison des pluies, alors qu'en surface, les maximums sont observés pendant la saison sèche ou des pluies selon la baie (Figure 6).
Les fortes teneurs en orthophosphates correspondent à la saison des crues en surface comme en profondeur dans toutes les baies. Les nitrites et l'ammonium ont accusé des teneurs moyennes plus élevées en saison des crues et des pluies (Figure 7). 




Figure 4 : Variations temporelles des ordres de grandeurs de l'ammonium aux stations 5 et 6 de la baie de Cocody, et 15 de la baie de Biétri.

\section{DISCUSSION}

Les valeurs moyennes extrêmes de température mesurées au cours de nos campagnes se situent dans la fourchette habituelle $\left(28,2\right.$ à $29,3{ }^{\circ} \mathrm{C}$ en surface et 25 à $29{ }^{\circ} \mathrm{C}$ au fond) (Pagès et al., 1979; Dufour 1982; Kouassi et al., 2005). Les gradients thermiques ont pour origines la coexistence en surface d'eaux continentales chaudes avec les eaux marines froides au fond, et la forte turbidité des eaux qui empêche la pénétration des rayons lumineux. Les fortes températures enregistrées pourraient augmenter la vitesse des réactions biologiques et biochimiques.

Les valeurs du $\mathrm{pH}$ et leurs variations spatiales observées peuvent s'expliquer par l'influence de plusieurs facteurs. En effet, les valeurs élevées pourraient s'expliquer par la prépondérance des eaux marines comme cela a été suggéré par Arfi et Guiral (1994), à l'activité photosynthétique du phytoplancton, et au caractère siliceux des bassins versants de la lagune (Debyser, 1955). En revanche, la proportion élevée d'eau fluviale ou météorologique, et les processus de dégradation et de minéralisation des particules organiques sont responsables des faibles valeurs enregistrées. Le $\mathrm{pH}$ est un facteur limitant dans les écosystèmes aquatiques : si le $\mathrm{pH}$ est inférieur à 4,5 ou supérieur à 10 , le milieu devient toxique pour les organismes vivants. Les valeurs moyennes de $\mathrm{pH}$ enregistrées au cours de cette étude (7,46 à 8,17 ) se situent dans la fourchette des $\mathrm{pH}$ normaux.

La salinité est un marqueur de l'origine des eaux. Les valeurs moyennes maximales de salinité observées dans les baies proches du canal de Vridi (Biétri et Milliardaires) s'expliquent par l'effet des eaux salées marines. La gamme des valeurs moyennes de salinité de cette étude est en accord avec celles observées dans la littérature $(13,86$ à 18,92\%o) (Varlet, 1978 ; Kouassi et al., 2005)

La transparence indique relativement le degré de pollution et permet de suivre la trajectoire d'un effluent dans le milieu récepteur (Pagès et al., 1980). Ainsi, les faibles valeurs obtenues dans les baies de Marcory, de Cocody, du Banco et de Biétri sont imputables aux apports élevés des eaux usées domestiques et industrielles. En revanche, les valeurs élevées observées en baie des Milliardaires indiquent que ce plan d'eau est soumis aux rejets urbains à un moindre degré. Les pluies locales et les eaux du fleuve Comoé contribuent à l'abaissement de la transparence des eaux par les apports de particules en suspension. Les faibles taux d'oxygène mesurés peuvent s'expliquer par les apports en grandes quantités de matières organiques par les rejets domestiques et 


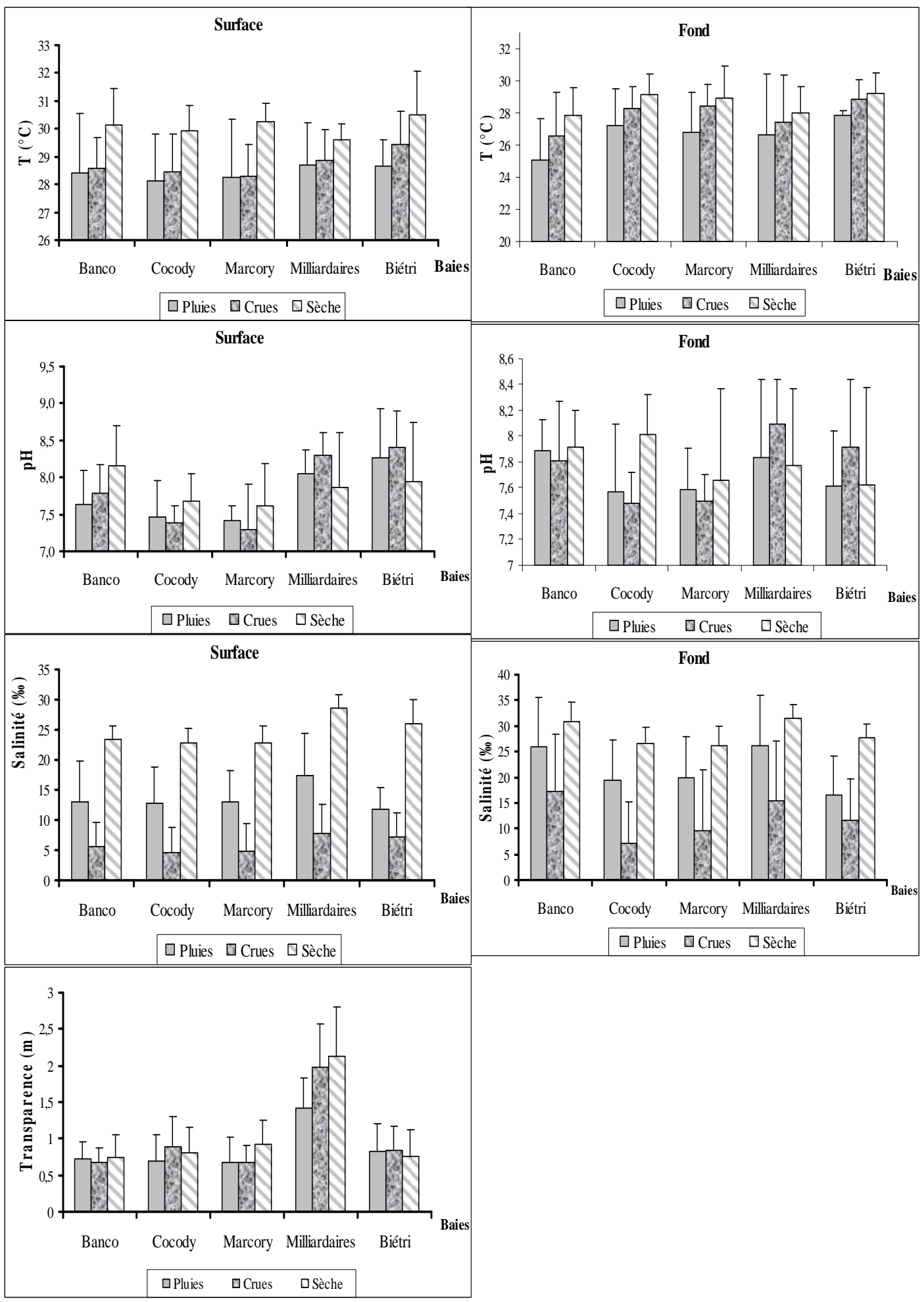

Figure 5 : Variations saisonnières de la température, du $\mathrm{pH}$, de la salinité à $0,5 \mathrm{~m}$ de la surface et du fond, et de la transparence des baies de la lagune Ebrié. Les barres verticales représentent les écarts types. 


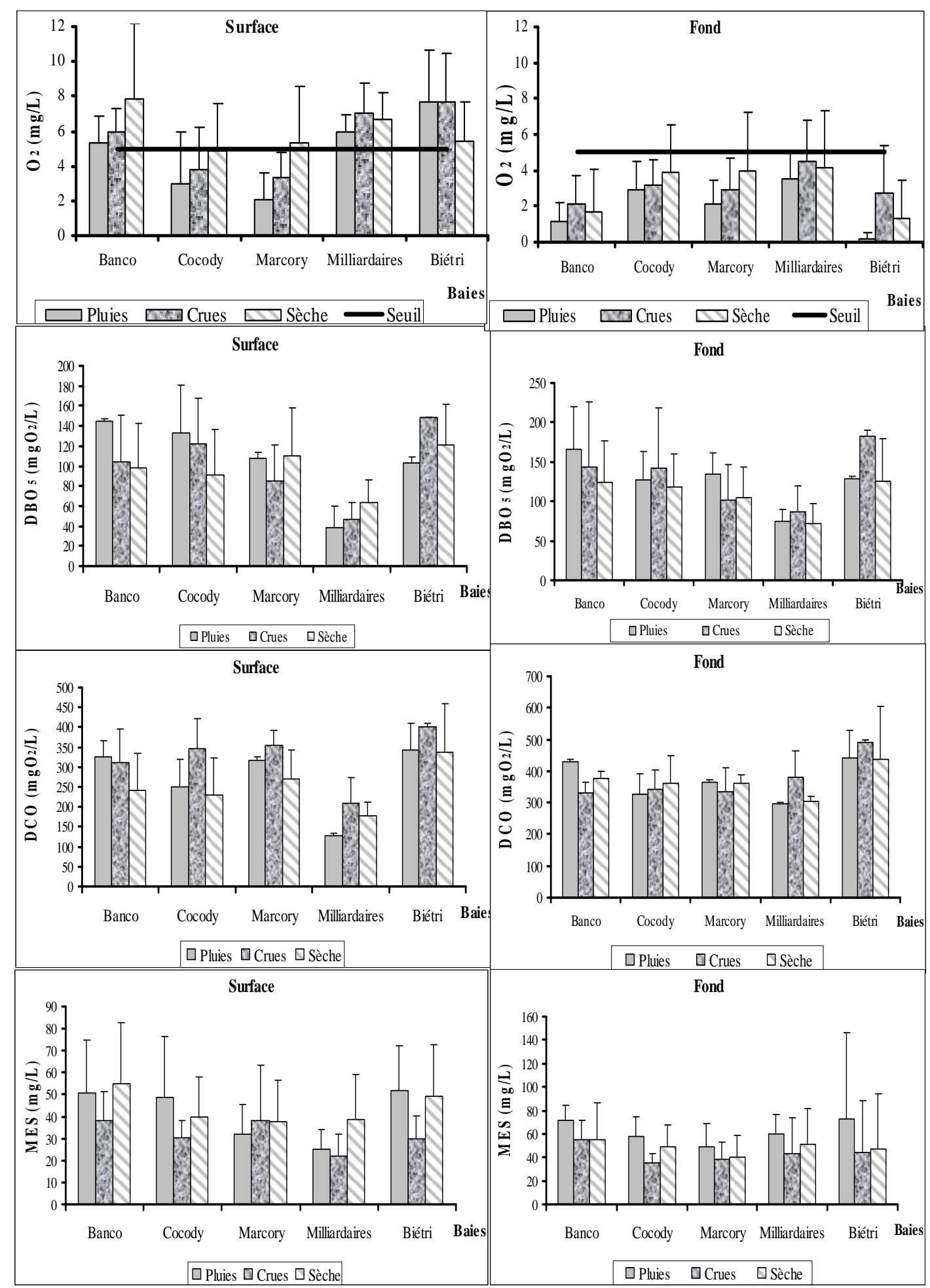

Figure 6 : Variations saisonnières de l'oxygène dissous, la $\mathrm{DBO}_{5}$, la $\mathrm{DCO}$ et des $\mathrm{MES}$ à $0,5 \mathrm{~m}$ de la surface et du fond des baies de la lagune Ebrié. Les barres horizontales représentent le seuil admis pour la bonne santé ( $5 \mathrm{mg} / \mathrm{l})$ d'un système aquatique. Les barres verticales représentent les écarts types. 

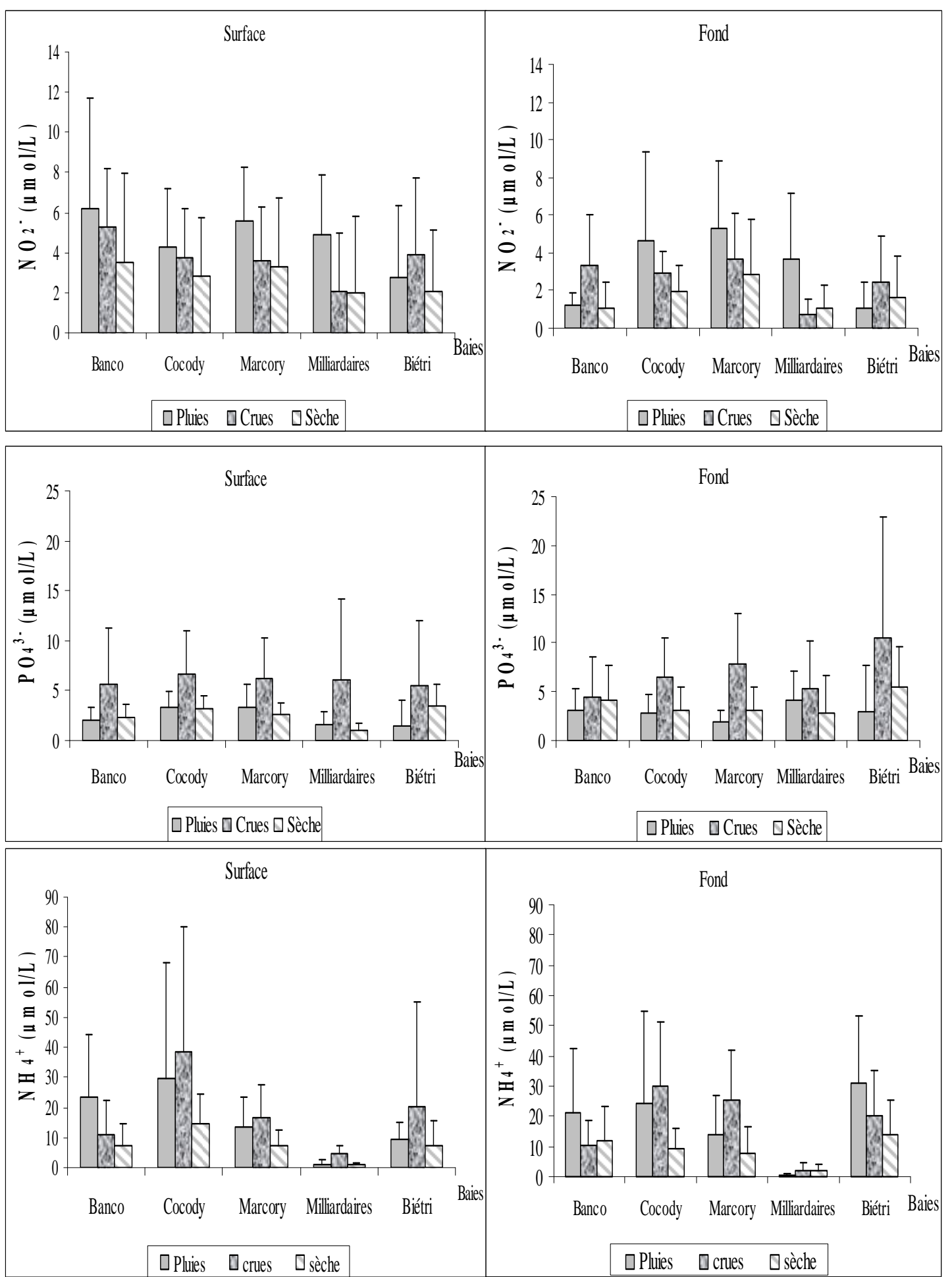

Figure 7 : Variations saisonnières des sels nutritifs à $0,5 \mathrm{~m}$ de la surface et du fond des baies de la lagune Ebrié. Les barres verticales représentent les écarts types. 
industriels ainsi que les eaux de ruissellement et l'érosion, le faible brassage des eaux, la nitrification des minéraux apportés dans les baies, le faible ensoleillement notamment en saison des pluies, et la remise en suspension des sédiments. En revanche, les sursaturations en oxygène observées résultent d'une part des apports d'eaux marines et de l'action des marées qui diluent l'eau des baies, et d'autre part de l'activité photosynthétique des algues et des apports atmosphériques d'oxygène par diffusion (Debyser, 1955). Au regard des seuils d'oxygène dissous au fond des milieux estuariens $(5 \mathrm{mg} / \mathrm{L})$, nous pouvons dire que la baie des Milliardaires peut être le lieu de stress et de réactions d'évitement de la faune aquatique contrairement aux autres baies étudiées où selon Pihl et al. (1992), Howell et Simpson (1994) et ERM (2000), il y a des mortalités plus ou moins massives de la faune. Ces hypothèses pourraient justifier les mortalités importantes de poissons constatées au cours de nos campagnes de février à avril 2008. En effet, selon Guiral et Chantraine (1983) et Guiral et Etien (1994), les mortalités notables de poissons en lagune Ebrié seraient dues aux fortes désoxygénations liées à la mort et à la décomposition très rapide de la biomasse phytoplanctonique et des macrophytes flottantes. Si l'on se réfère aux critères de qualités établies par Beaupoil et Bornens (1997), la baie des Milliardaires est de qualité moyenne tandis que les autres baies sont de qualité hors classe niveau 1.

Karuppanapandian et al. (2007) ont rapporté des valeurs maximales de $\mathrm{DBO}_{5}$ de 8,29 mg/L en Inde, dans la baie de Palk. De plus, Dufour (1982) a relevé une médiane maximale de $8 \mathrm{mg} / \mathrm{L}$ au fond de la baie de Biétri. Pagès et al. (1980) ont relevé des valeurs de DCO allant de 50 à $200 \mathrm{mg} / \mathrm{L}$ dans le bassin du port. Les ordres de grandeur de la $\mathrm{DBO}_{5}$ et de la DCO enregistrés dans cette étude sont largement supérieurs à ces valeurs, ce qui est le fait de la croissance des apports de composés organiques biodégradables ou non par les effluents domestiques et industriels en lagune sans traitement préalable. En effet, les valeurs moyennes de $\mathrm{DBO}_{5}$ et de DCO trouvées dans toutes les baies soumises à des rejets directs sont 2 à 3 fois plus élevées que celles de la baie des Milliardaires. De plus Yao et al. (2007) ont obtenue des moyennes de $\mathrm{DBO}_{5}$ allant de 600

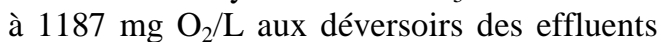
domestiques et industriels dans les baies de Biétri, Cocody et Marcory. Un autre facteur non négligeable est l'envahissement des eaux lagunaires par les macrophytes flottantes lors des crues du fleuve Comoé (Amon et al., 1991) qui sans équivoque, augmente significativement la demande en oxygène. Les teneurs en phytoplancton et en chlorophylle sont d'autres importantes sources de demande en oxygène (Heiskary et Markus, 2001; MacPherson, 2003). A l'interface eausédiment, le milieu est réducteur; ce qui est favorable à la préservation de la matière organique donc de la forte demande en oxygène. $\mathrm{La} \mathrm{DBO}_{5}$ n'a pas la valeur absolue qu'on lui prête parfois. Bien qu'il n'existe pas actuellement de taux admissibles ou recommandables de $\mathrm{DBO}_{5}$ pour la qualité des eaux lagunaire, nous pouvons néanmoins affirmer que les baies de Cocody, Banco, Marcory et de Biétri sont l'objet d'une forte pollution par les matières oxydables.

Les valeurs des matières en suspensions confirment les observations faites sur la transparence des eaux. Le pic des moyennes de transparence mesuré en baie des Milliardaires coïncide avec le minimum des MES. Les concentrations moyennes en MES déterminées dans les baies s'inscrivent dans la fourchette des valeurs signalées dans la littérature par certains auteurs pour l'ensemble de la lagune Ebrié (20 à $50 \mathrm{mg} / \mathrm{L}$ ) (Lemasson et al., 1981, Guiral et al., 1993). Elles restent cependant très inférieures aux résultats de Tastet (1974) (valeurs comprises entre 10 et $400 \mathrm{mg} / \mathrm{L}$ avec une moyenne de $90 \mathrm{mg} / \mathrm{L}$ ) et de Kouassi et al. (2005) de 1987 à 1988 (48 à $97,04 \mathrm{mg} / \mathrm{L}$ en surface et 128 à $151,60 \mathrm{mg} / \mathrm{L}$ au fond). Ces données paraissent très variables d'une étude à l'autre et d'un site à l'autre et reflètent l'hétérogénéité du bassin versant drainé et des rejets qui se déversent en lagune. Cependant, la disparité des résultats observés peut également s'expliquer par des différences dans les techniques d'échantillonnage et d'analyse.

La forte teneur en matière organique des particules en suspension est liée à la nature des rejets domestiques et industriels et des particules apportées en lagune par les eaux de ruissellement (Guiral et al., 1993) et au 
mode d'occupation du sol. Les résultats suggèrent d'une part une accumulation de sédiments riches en matières organiques dans les baies du Banco, Cocody, Marcory et Biétri et d'autre part, un dépôt de particules minérales dans les fonds de la baie des Milliardaires. Les matières en suspension de natures minérales enregistrées en baie des Milliardaires pourraient être le fait de l'érosion des sols du bassin versant et des forts ruissellements qui entraînent les sables dans ce plan d'eau.

Dans les baies proches des rejets urbains industriels, la forme particulaire est très forte, ce qui est reléguable aux déversements abusifs sans traitement préalable. Le ruissellement et le lessivage des sols augmentent cette pollution lors de la saison des pluies. La biomasse algale très élevée constitue une autre source (jusqu'à 100 $\mathrm{mg} / \mathrm{m}^{3}$ de chlorophylle a) (Arfi et al., 1994). Il semble que les eaux marines diminuent fortement l'intensité de la pollution organique particulaire par dilution.

Les concentrations en nitrites généralement plus faibles atteignant parfois des valeurs nulles à l'interface eau-sédiment dans les secteurs profonds peuvent s'expliquer par leur réduction en azote ammoniacal dans les conditions anaérobies qui règnent dans ces couches. L'azote ammoniacal, issu de la dégradation anaérobie par les microorganismes de la matière organique accumulée dans la colonne d'eau et dans les sédiments, présente de fortes teneurs dans la couche profonde. Les niveaux les plus élevés pour tous les sels observés en saison des crues et des pluies montrent que les eaux du fleuve Comoé en crue, les eaux de ruissellement et l'érosion des sols du bassin versant sont les sources majeures de sels nutritifs en lagune Ebrié.

Selon Agences de 1'Eau et Ministère de l'Environnement (1994), la vie aquatique peut être gravement atteinte pour des concentrations en azote ammoniacal de l'ordre de $111 \mu \mathrm{mol} / \mathrm{L}(2 \mathrm{mg} / \mathrm{L})$ à un $\mathrm{pH}$ de 7,4 à 8,5. Les valeurs moyennes des baies tombent en dessous de cette valeur, ce qui soulève l'hypothèse d'une bonne santé de ces plans d'eau pour ce paramètre. Cependant, les zones à faibles taux de renouvellement et soumises à de fortes quantités d'eaux usées et industrielles (stations 5,6 et 15), ont présenté des concentrations d'ammonium supérieures à ce seuil, avec une tendance à la hausse durant la période d'étude. En outre, la comparaison des ordres de grandeurs d'ammonium mesurés en baie de Biétri et des Milliardaires révèle que la moyenne de la baie de Biétri vaut 13 fois celle des Milliardaires. Ces résultats traduisent l'impact des rejets urbains sur la santé de ces plans d'eau.

Les teneurs en ammonium des eaux de ces campagnes s'avèrent très élevées par rapport à celles obtenues par Kouassi et al. (2005) dans les mêmes baies de janvier 1993 à décembre 1998 (4,4 à $16 \mu \mathrm{mol} / \mathrm{L})$. Cette augmentation des teneurs en ammonium peut s'expliquer par l'urbanisation accélérée, l'usage croissant des engrais et la croissance démographique et industrielle.

\section{Variations saisonnières}

Les variations saisonnières des températures superficielles observées sont typiques du climat équatorial (Garcia, 1972 ; Varlet, 1978); la saison des pluies est caractérisée par des températures faibles dues au refroidissement thermique de l'air troposphérique tandis que la saison sèche correspond aux valeurs maximales (Arfi et Guiral, 1994). Les faibles salinités observées pendant la saison des crues et des pluies s'expliquent par la prépondérance des eaux continentales douces et de pluies en lagune tandis que les fortes salinités résultent de l'importance d'eaux salées marines (valeurs moyennes > 35\%o) (Varlet, 1978).

La nette décroissance des concentrations d'oxygène dissous en saison des pluies est attribuable d'une part, à la forte turbidité des eaux qui provoque une baisse de la production photosynthétique, et d'autre part, à l'oxydation des grandes quantités de matières organiques charriées en lagune. Les fortes valeurs de demande en oxygène observées pendant les saisons des pluies et des crues peuvent être mises sur le compte des apports par le réseau d'assainissement pluvial de volumes d'eaux très importants, fortement chargés de matériaux et détritus accumulés sur les voies, de résidus des eaux usées domestiques et des eaux de lessivage des latrines. Nos résultats corroborent ceux de Dufour (1982) qui ont indiqué des $\mathrm{DBO}_{5}$ 
maximales pendant les saisons des crues et des pluies.

La forte évaporation des eaux qui s'opère en lagune pendant la saison sèche et la prolifération planctonique contribuent à augmenter les charges de particules en suspension dans les couches superficielles. Guiral et al. (1993) ont observé des teneurs de MES plus importantes $(52,3 \mathrm{mg} / \mathrm{L})$ en saison sèche, ce qui corrobore nos observations. Pendant la saison des pluies, l'intensité des apports de solides en suspension par les eaux de ruissellement et les effluents domestiques industriels est maximale ; mais il semble avoir un fort taux de sédimentation dû à la faible densité des eaux (essentiellement douces) qui diminue la quantité de MES de la couche de surface au profit de l'hypolimnion. Il en résulte de forte concentrations de MES à l'interface eau-sédiment.

Les niveaux les plus élevés pour tous les sels observés en saison des crues et des pluies montrent que les eaux du fleuve Comoé en crue, les eaux de ruissellement et l'érosion des sols du bassin versant, et les effluents domestiques et industriels d'Abidjan sont les sources majeures de sels nutritifs en lagune Ebrié.

\section{Relations entre les paramètres de pollution}

Une analyse de corrélation de Pearson effectuée entre les différents paramètres de pollution montre que la charge organique particulaire (MVS) est significativement liée aux matières en suspension $(r=0,80$ en surface et $r=0,77$ au fond). Ceci indique que les fortes teneurs de MES en lagune contribuent à l'enrichissement des eaux en matières organiques. Aucune corrélation n'a été observée entre l'oxygène dissous vs la $\mathrm{DBO}_{5}$, la DCO et les MES (Tableaux 2 et 3); ce qui serait attribuable à l'effet des différents facteurs intervenant dans le bilan d'oxygène dissous dans les eaux de la lagune.

\section{Conclusion}

Les valeurs mesurées pour la température, le $\mathrm{pH}$, la salinité, la conductivité au cours de ces campagnes se situent dans la fourchette habituelle. L'impact de la quantité abondante des eaux usées domestiques et industrielles qui se déversent dans les baies du Banco, Cocody, Marcory, et Biétri a été mis en évidence par de faibles valeurs de transparence et des valeurs moyennes de $\mathrm{DBO}_{5}$ et de DCO 2 à 3 fois plus élevées que celle de la baie des Milliardaires. En outre, les particules apportées en lagune sont très chargées en matière organique, ce qui a engendré plusieurs foyers d'hypoxies et d'anoxies dans ces plans d'eaux. Les teneurs en oxygène dissous et en ammonium révèlent que les quatre premières baies sont des zones où l'on pourrait observer d'importantes mortalités de la faune.

La dépollution des baies urbaines de la lagune Ebrié est devenue une nécessité.

Tableau 2: Coefficients de corrélation de Pearson entre les différents paramètres physicochimiques mesurés (2006-2008) à la surface des eaux de la lagune Ebrié.

\begin{tabular}{|c|c|c|c|c|c|c|c|c|c|c|c|}
\hline Surface & DCO S & DBO S & MES & MVS & $\mathbf{T}^{\circ} \mathbf{C}$ & pH & Salinité & $\mathbf{O}_{2}$ & $\mathrm{NO}_{2}^{-}$ & $\mathbf{N H}_{4}^{+}$ & $\mathrm{PO}_{4}^{3-}$ \\
\hline $\mathrm{DCO}$ & 1 & & & & & & & & & & \\
\hline DBO & $0,48 * *$ & 1 & & & & & & & & & \\
\hline MES & 0,19 & 0,03 & 1 & & & & & & & & \\
\hline MVS & 0,09 & 0,03 & $0,80 *$ & 1 & & & & & & & \\
\hline $\mathrm{T}^{\circ} \mathrm{C}$ & $-0,13$ & $-0,19$ & 0,08 & $-0,01$ & 1 & & & & & & \\
\hline $\mathrm{pH}$ & $-0,04$ & $-0,05$ & 0,03 & $-0,04$ & 0,23 & 1 & & & & & \\
\hline Salinité & $-0,20$ & $-0,08$ & 0,25 & $-0,06$ & $0,53 * *$ & 0,17 & 1 & & & & \\
\hline $\mathrm{O}_{2}$ & $-0,04$ & 0,03 & $-0,01$ & $-0,03$ & 0,20 & $0,50 *$ & 0,07 & 1 & & & \\
\hline $\mathrm{NO}_{2}^{-}$ & 0,09 & 0,28 & 0,11 & 0,04 & $-0,31$ & $-0,24$ & $-0,34 * *$ & $-0,18$ & 1 & & \\
\hline $\mathrm{NH}_{4}^{+}$ & 0,13 & 0,11 & $-0,07$ & 0,02 & $-0,09$ & $-0,02$ & $-0,30 * *$ & $-0,15$ & 0,11 & 1 & \\
\hline $\mathrm{PO}^{3-}{ }_{4}$ & $-0,08$ & $-0,14$ & $-0,24$ & 0,12 & 0,09 & $-0,05$ & $-0,47 * *$ & $-0,14$ & 0,09 & 0,27 & 1 \\
\hline
\end{tabular}


Tableau 3: Coefficients de corrélation de Pearson entre les différents paramètres physicochimiques mesurés (2006-2008) sur 160 mesures à l'interface eau-sédiment des eaux de la lagune Ebrié.

\begin{tabular}{|c|c|c|c|c|c|c|c|c|c|c|c|}
\hline Fond & DCO & DBO & MES & MVS & $\mathbf{T}^{\circ} \mathbf{C}$ & pH & Salinité & $\mathrm{O}_{2}$ & $\mathrm{NO}_{2}^{-}$ & $\mathrm{NH}_{4}^{+}$ & $\mathrm{PO}^{3-}{ }_{4}$ \\
\hline $\mathrm{DCO}$ & 1 & & & & & & & & & & \\
\hline DBO & $0,50 * *$ & 1 & & & & & & & & & \\
\hline MES & 0,16 & 0,01 & 1 & & & & & & & & \\
\hline MVS & $-0,06$ & 0,13 & $0,77 *$ & 1 & & & & & & & \\
\hline $\mathrm{T}^{\circ} \mathrm{C}$ & $-0,11$ & $-0,02$ & $-0,29$ & 0,20 & 1 & & & & & & \\
\hline $\mathrm{pH}$ & $-0,07$ & $-0,05$ & 0,02 & $-0,05$ & 0,03 & 1 & & & & & \\
\hline Salinité & 0,10 & $-0,10$ & $0,33 * *$ & $-0,26$ & $-0,25$ & 0,18 & 1 & & & & \\
\hline $\mathrm{O}_{2}$ & $-0,15$ & $-0,14$ & $-0,36^{* *}$ & $-0,30 * *$ & 0,17 & $0,30 * *$ & $-0,16$ & 1 & & & \\
\hline $\mathrm{NO}_{2}^{-}$ & 0,07 & 0,15 & $-0,02$ & 0,03 & $-0,05$ & $-0,27$ & $-0,34 * *$ & $-0,07$ & 1 & & \\
\hline $\mathrm{NH}_{4}^{+}$ & 0,03 & 0,18 & $-0,04$ & 0,16 & 0,07 & $-0,29$ & $-0,39 * *$ & $-0,18$ & 0,14 & 1 & \\
\hline $\mathrm{PO}_{4}^{3-}$ & 0,03 & 0,11 & $-0,09$ & 0,15 & 0,22 & $-0,14$ & $-0,34 * *$ & $-0,16$ & 0,05 & 0,12 & 1 \\
\hline
\end{tabular}

\section{REFERENCES BIBLIOGRAPHIQUES}

Agences de l'Eau et Ministère de l'Environnement. 1994. L'assainissement des agglomérations: techniques d'épuration actuelles et évolutions. Cahier Technique France, 27: 89-90.

Aminot A, Chaussepied M. 1983. Manuel des Analyses Chimiques en Milieu Marin. CNEXO, Editions Jouve ; 395.

Amon KJP, Guiral D, Sankaré Y, Kaba N, Etien N.1991. Suivi cartographique de l'expansion des macrophytes envahissant le système lagunaire Ebrié. J. Ivoir. Oéanol. Limnol. Abidjan, 1(2): 11-24.

Arfi R, Guiral D. 1994. Un écosystème estuarien eutrophe: la baie de Biétri. In Environnement et Ressources Aquatiques de Côte d'Ivoire, Durand JR, Dufour P, Guiral D, Zabi S (éds). Tome II - Les milieux lagunaires. Editions de l'ORSTOM ; 335-363.

Arfi R, Dufour P, Maurer D. 1981. Phytoplancton et pollution: première étude en baie de Biétri (Côte d'Ivoire). Traitement mathématique des données. Oceanol. Acta., 4: 319330.

Beaupoil C, Bornens P. 1997. Oxygène Dissous et Toxicité de l'Ammoniaque en Zones Estuariennes: Seuils d'Acceptabilité. Station de Biol. Mar. (Concarneau). Ed. Agence de l'Eau Loire-Bretagne; 48.
Centre d'Expertise en Analyse Environnementle du Quebec. 2003. Détermination de la demande biochimique en oxygène dans les effluents: méthode électrométrique. MA.315-DBO 1.O, Ministère de l'Environnement du Québec, 14.

Debyser J. 1955. Etude sédimentologique du système lagunaire d'Abidjan (Côte d'Ivoire). Rev. Inst. Fr. Pétrole, 1(5): 319-334.

Dufour P. 1982. Les frontières naturelles et humaines du système lagunaire Ebrié. Incidence de l'hydroclimat. Hydrobiologia, 94: 105-120.

Dufour P, Slépoukha M. 1975. L'oxygène dissous en lagune Ebrié: influence de l'hydroclimat et des pollutions. Doc. Sci. Centre Rech. Océangr. Abidjan, 6(2):75118.

Durand JR, Skubich M. 1979. Recherches sur les lagunes ivoiriennes. Doc. Centre Rech. Océanogr. Abidjan, 10: 55.

ERM. 2000. Criteria used for the definition of eutrophication in marine/coastal waters. European Commission, D. G. Environment, 71.

Garcia S, 1972. Biologie de Penaeus duorarum (Burk.) en Côte d'Ivoire. II. Ponte et migration. Doc. Centre Rech. Oceanogr. Abidjan 3: 19-45.

Guiral D, Arfi R, Torreton JP. 1984. Devenir de la matière organique particulaire dans 
un milieu eutrophe tropical. Rev. Hydrobiol. Trop., 17: 191-206.

Guiral D, Chantraine JM. 1983. Hypothèses sur l'origine des mortalités observées en lagune Ebrié en 1979. Doc. Centre Rech. Oceanogr. Abidjan, 14: 61-95.

Guiral D, Etien N. 1994. Les macrophytes. In Environnement et Ressources Aquatiques de Côte d'Ivoire. Tome II Les Milieux Lagunaires, Durand JR, Dufour P, Guiral D, Zabi S (eds). Editions de l'ORSTOM ; 137-154.

Guiral D, Kouassi AM, Arfi R. 1993. Estimation des niveaux de pollution organique et bactérienne des eaux à proximité des berges de la ville d'Abidjan (Lagune Ebrié- Côte d'Ivoire). J. Ivoir. Océanol. Limnol., 2(1): 1-18.

Haskoning. 1999. Dépollution de la lagune Ebrié, Abidjan. Mission de reconnaissance, $37 \mathrm{p}$.

Heikskary S, Markus H. 2001. Establishing relationships among nutrient concentrations, phytoplankton abundance, and biochemical oxygen demand in Minnesota, USA, rivers. Journal of Lake and Reservoir Management, 17: 251-267.

Howell P, Simpson D. 1994. Abundance of marine resources in relation to dissolved oxygen in Long Island Sound. Estuaries, 17(2): 394-402.

Karuppanapandian T, Karuppudurai T, Kumaraguru AK. 2007. A preliminary study on the environmental condition of the coral reef habitat. Int. J. Environ. Sci. Tech., 4: 371- 378.

Koroleff F. 1976. Determination of ammonia, In Methods of Sea Water Analysis, Grasshoff K (éd). Verlag Chemie, Wenheim: RFA; 126-133.

Kouassi AM, Tidou AS, Kamenan A. 2005. Caractéristiques hydrochimiques et microbiologiques des eaux de la lagune Ebrié (Côte d'Ivoire). Partie I : Variabilité saisonnière des paramètres hydrochimiques. Agron. Afr., 17(2): 73162.

Lemasson L, Pages J, Dufour P, Cremoux JL. 1981. Matière organique particulaire et biomasse dans une lagune tropicale. Rev.
Hydrobiol. Trop., 13(3): 191-212.

Mac Pherson TA. 2003. Sediment oxygen demand and biochemical oxygen demand: patterns of oxygen depletion in tidal creek study sites. M. S. Thesis, the University of North Carolina at Wilmington, Wilmington, NC. 55.

Murphy J, Riley JP. 1962. A modified single solution method for the determination of phosphate in natural waters. Anal. Chim. Acta, 27: 31-36.

Pagès J, Dufour P, Lemasson L. 1980. Pollution de la zone urbaine de la lagune Ebrié (Côte d'Ivoire). Doc. Sci. Centre Rech. Océanogr., Abidjan, 11(2): 79-107.

Pagès J, Lemasson L, Dufour P. 1979. Eléments nutritifs et production primaire dans les lagunes de Côte d'Ivoire. Cycle annuel. Arch. Sci. Centre Rech. Océanogr. Abidjan, 5:1-60.

Pihl L, Baden SP, Diaz RJ, Schaffner LC. 1992. Hypoxia-induced structural changes in the diet of bottom-feeding fish and crustacea. Marine Biology, 112: 349361.

Rodier J. 1996. L'Analyse de l'Eau (8 $8^{\mathrm{eme}}$ édn). Dunod, Paris: France.

Tastet JP. 1974. L'environnement physique du système lagunaire Ebrié. Université d'Abidjan. Fac. Sci. Dept. Sci. Terre, Série Documentation, 11: 28.

Varlet F. 1978. Le régime de la lagune Ebrié (Côte d'Ivoire). Traits physiques essentiels, Trav. Doc. ORSTOM 83, p.164.

Winkler L.W. 1888. Die Bestimmung des in wasser gelosten Seuerstoffes. Chem. Ber., 21: 2843-2855.

Yao KM, Métongo BS, Trokourey A, Bokra Y. 2007. Détermination de certains paramètres de pollution dans les baies d'une lagune tropicale: la lagune Ebrié (Côte d'Ivoire). J. Ivoir. Océanol. Limnol., 4(1): 1-10.

Zabi SG, 1982. Les peuplements benthiques liés à la pollution en zone urbaine d'Abidjan (Côte d'Ivoire). Actes Symposium international sur les lagunes côtières, Bordeaux 8-14 septembre 1981. Oceanologica Acta, suppl. 4: 441-455. 